\title{
Design and Synthesis of Some New Derivatives of Chlorobenzyl-Oxy-Phenyl-Ethyl-Thio-1H-Tetrazole and Study Their Antibacterial and Antifungal Activity
}

Ajay N Ambhore ( $\sim$ ambhoreajay@gmail.com )

Padmabhushan Dr Vasantraodada Patil Mahavidyalay Tasgaon https://orcid.org/0000-0002-09932440

\section{Research Article}

Keywords: Tetrazole, Bleaching Earth Clay (BEC), PEG-400, Antibacterial, Antifungal

Posted Date: December 20th, 2021

DOI: https://doi.org/10.21203/rs.3.rs-1173153/v1

License: (c) (1) This work is licensed under a Creative Commons Attribution 4.0 International License.

Read Full License 


\title{
Design and synthesis of some new derivatives of chlorobenzyl-oxy-phenyl- ethyl-thio-1H-tetrazole and study their antibacterial and antifungal activity
}

\author{
Ajay N. Ambhore* \\ *Padmabhushan Dr. Vasantraodada Patil Mahavidyalaya, Tasgaon. Dist. Sangli (MS) India \\ (Affiliated to Shivaji University, Kolhapur) \\ Email: ambhoreajay@gmail.com
}

\begin{abstract}
In recent years tetrazole scaffolds have been attracted interest in the field of synthetic and medicinal chemistry research. The unique structure of the tetrazole derivatives exhibits widespread applications in biology and technology. The close structural resemblance with carboxylic acid acts as a booster of the latter. Due to this diversified potential utilization, several methods are reported for the synthesis of tetrazole scaffolds.

Here in this chapter, we describe the synthesis of chlorobenzyl-oxy-phenyl-ethyl-thio1H-tetrazole derivatives (6a-p). The newly synthesized derivatives are characterized by spectral characterization and screened for their antifungal activity. Among these, some of the newly synthesized compounds show potent antifungal activity.

Keywords: Tetrazole, Bleaching Earth Clay (BEC), PEG-400, Antibacterial, Antifungal

\section{Introduction:}

Escalation of multidrug resistance strain, the evolution of incurable micro-organism, and gain in pathogenic microbes alarm the seriousness of microbial infection [1-3].To oversight this, researchers are uniformly focused on the synthesis of influential and adequate antimicrobial agents. Numerous antimicrobial scaffolds are disclosed and applied extensively for the treatment, prevention, and control of microbial infection. But still, there are some problems like, adverse
\end{abstract}


effects, high toxicity, and narrow antimicrobial spectrum which are unresolved. Literature in recent years endorsed that, the total number of publications on the chemistry of N-containing heterocycles consequential boost to discover the new drugs and assuring their biological activity which mainly contains tetrazole moiety.

Tetrazole is an important heterocyclic scaffold containing nitrogen. It is a unique nitrogen-rich compound among the known stable heterocycles and in spite not found in nature [4]. In 1885, for the first time synthesized and characterized compound embedding a tetrazole ring, is 2-phenyl-2H-tetrazole-5-carbonitrile [5]. Tetrazole is a remarkable synthetic scaffold that noticed wide applications in various fields like in medicinal, pharmacological, biochemistry, and in industrial [6-10].

Tetrazole is the lead motif in the field of medicinal chemistry. The uncommon, polynitrogen, electron-rich planer structure of the tetrazole ring is answerable to exhibit widespread applications for the treatment of various diseases especially antimicrobial [11-12], antifungal [13-14], antiviral [15-16], antitubercular [17-18], anticancer [19-20], antimalarial [21-22], antiangiogenic [23-24], anti-inflammatory [25-26], anti-HIV [27-28], analgesic [29-30], antiAlzheimer [31-32], antinociceptive [33-34], and anticonvulsant [35-36]. Weak interactions like hydrogen bonding, van der wals force, or coordination bonding are responsible for binding the tetrazole ring easily with the various enzymes or receptors of microbes and are successfully applied as components for comprehensive pharmaceutical applications [37]. Food and Drug Administration (FDA) approved several drugs possessing tetrazole scaffold as an important pharmaceutical agent [38]. Metabolic stable surrogates for carboxylic acid group and lipophilic spacers of tetrazole derivatives make it important in drug design [39]. It has also relived that the 
lipophilic nature of tetrazole ring in a drug revamps its oral bioavailability and cell penetration [37].

Besides this, tetrazole has noticed a broad spectrum of applications in materials like information recording systems [40], rocket propellants [41-42], specialty explosives [43], and agrochemical applications [44-47]. As befitting coordination property, it has also capable to form nitrogen containing ligands and designing stable complex with disparate metal ions and react as precursors to varied nitrogen containing heterocyclic compounds in organic synthesis [48]. Thus, the whispered applications of tetrazole skeleton in numerous fields make interest for the development of novel tetrazole derivatives. Some of them are displayed in Fig. 1.1.

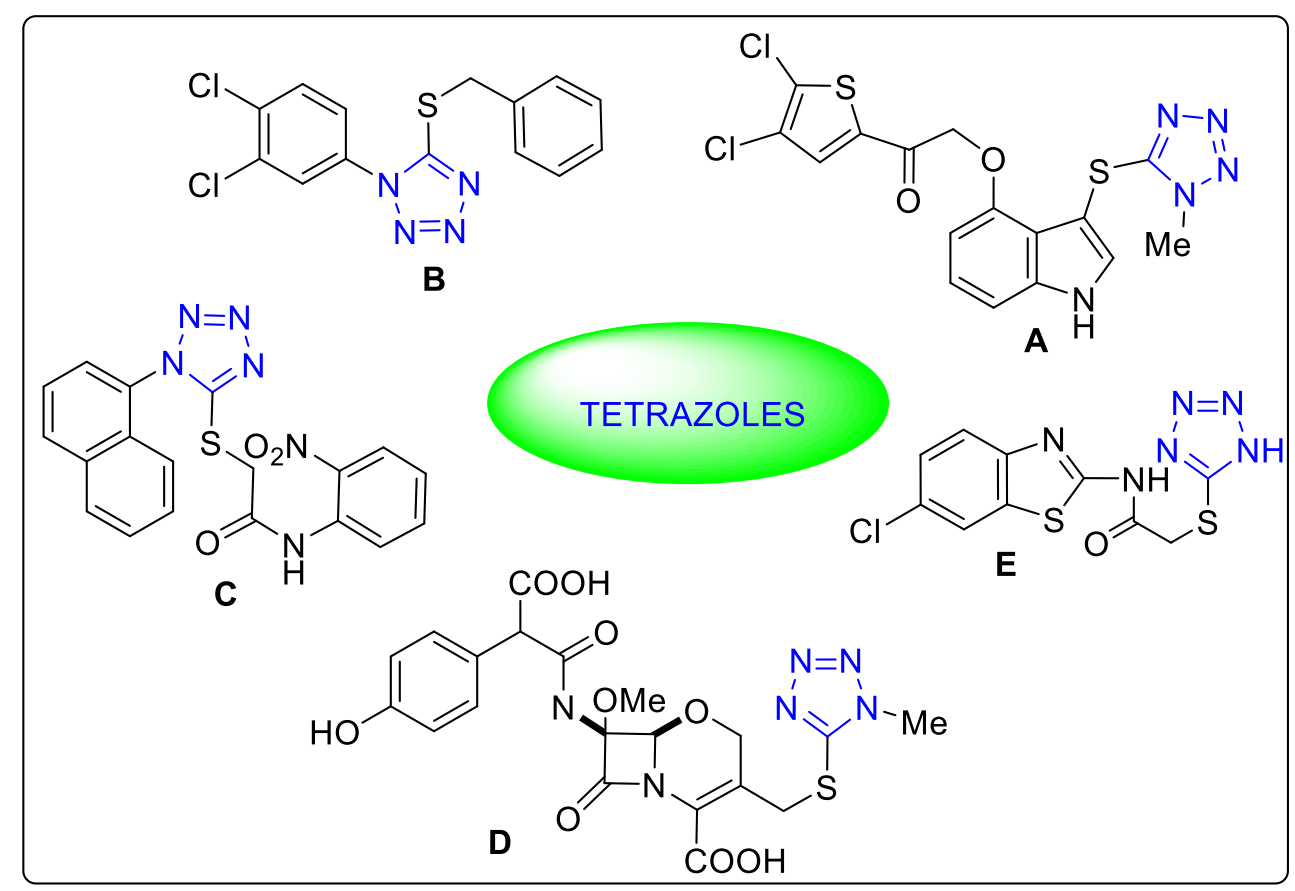

Fig 1. Some bioactive molecules bearing tetrazole ring.

By studying the above literature data and as a part of our research to design and synthesis bioactive heterocyclic compounds [49-53], in the present work, our strategy is to synthesize tetrazole embedded novel derivatives and study their antibacterial and antifungal activity. 


\section{Result and Discussion}

\section{Chemistry}

The synthetic pathway of the target compound chlorobenzyl-oxy-phenyl-ethyl-thio-1Htetrazole derivatives (6a-p) is summarized in Scheme 1

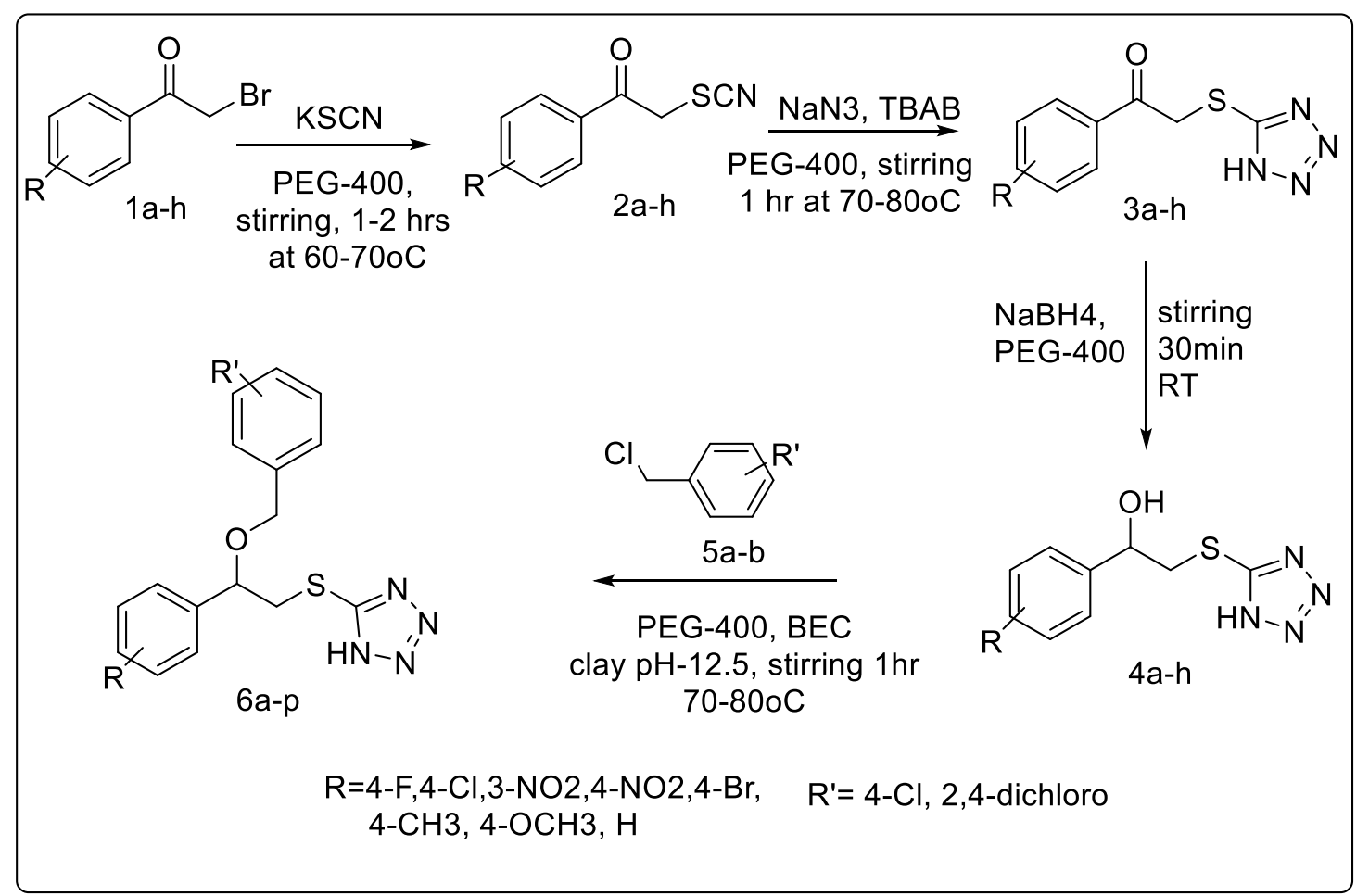

Scheme 1. Synthetic pathway of chlorobenzyl-oxy-phenyl-ethyl-thio-1H-tetrazole derivatives (6a-p)

This scheme involves a reaction between substituted phenacyl bromides (1a-h) with potassium thiocynate in PEG-400 to provide the thiocynated intermediate (2a-h). In turn compound (2a-h) was treated with sodium azide in presence of TBAB (Tetra Butyl Ammonium Bromide) as a phase transfer catalyst and PEG-400 to generate cyclized intermediate (3a-h). Further, the $\mathrm{C}=\mathrm{O}$ bond of the compound (3a-h) undergoes reduction by Sodium borohydride $\left(\mathrm{NaBH}_{4}\right)$ in PEG-400 at room temperature to yield the next intermediate products (4a-h). Then 
compounds (4a-h) were to couple with chlorobenzyl chloride (5a-b) by using catalytic amount BEC in PEG-400 to give the targeted compound chlorobenzyl-oxy-phenyl-ethyl-thio- $1 \mathrm{H}$ tetrazole derivatives (6a-p) in admirable yields (Scheme 2).<smiles>Fc1ccc(C(CSc2nnn[nH]2)OCc2ccc(Cl)cc2)cc1</smiles>

$6 a, 86 \%$<smiles>Clc1ccc(COC(CSc2nnn[nH]2)c2ccc(Cl)cc2)cc1</smiles>

$6 b, 89 \%$<smiles>Clc1ccc(COC(CSc2nnn[nH]2)c2ccc(Br)cc2)cc1</smiles>

$6 c, 90 \%$<smiles>O=[N+]([O-])c1ccc(C(CSc2nnn[nH]2)OCc2ccc(Cl)cc2)cc1</smiles>

$6 d, 92 \%$<smiles>O=[N+]([O-])c1cccc(C(CSc2nnn[nH]2)OCc2ccc(Cl)cc2)c1</smiles>

$6 e, 92 \%$<smiles>Cc1ccc(C(CSc2nnn[nH]2)OCc2ccc(Cl)cc2)cc1</smiles>

$6 f, 87 \%$<smiles>COc1ccc(C(CSc2nnn[nH]2)OCc2ccc(Cl)cc2)cc1</smiles>

$6 g, 82 \%$<smiles>Clc1ccc(COC(CSc2nnn[nH]2)c2ccccc2)cc1</smiles>

$6 h, 85 \%$<smiles>Fc1ccc(C(CSc2nnn[nH]2)OCc2ccc(Cl)cc2Cl)cc1</smiles>

$6 i, 84 \%$<smiles>Clc1ccc(C(CSc2nnn[nH]2)OCc2ccc(Cl)cc2Cl)cc1</smiles>

$6 \mathrm{j}, 85 \%$<smiles>Clc1ccc(COC(CSc2nnn[nH]2)c2ccc(Br)cc2)c(Cl)c1</smiles>

$6 \mathrm{k}, 86 \%$<smiles>O=[N+]([O-])c1ccc(C(CSc2nnn[nH]2)OCc2ccc(Cl)cc2Cl)cc1</smiles>

6I, $91 \%$<smiles>O=[N+]([O-])c1cccc(C(CSc2nnn[nH]2)OCc2ccc(Cl)cc2Cl)c1</smiles>

$6 \mathrm{~m}, 89 \%$<smiles>Cc1ccc(C(CSc2nnn[nH]2)OCc2ccc(Cl)cc2Cl)cc1</smiles>

$6 n, 86$<smiles>COc1ccc(C(CSc2nnn[nH]2)OCc2ccc(Cl)cc2Cl)cc1</smiles>

$6 \circ, 80 \%$<smiles>Clc1ccc(COC(CSc2nnn[nH]2)c2ccccc2)c(Cl)c1</smiles>

$6 p, 83 \%$ 
Scheme 2. Variation of substituent on tetrazole (6a-p)

To achieve the target compounds (6a-p), here we reported an eco-friendly synthetic route which accomplishes by using BEC ( $\mathrm{pH} 12.5)$ as a heterogeneous catalyst to attain the basic media in PEG-600 as a green reaction solvent.

Finally, the structures of the synthesized products were determined by IR, ${ }^{1} \mathrm{H}$ NMR, ${ }^{13} \mathrm{C}$ NMR, ESI-MS and elemental analyses data. The IR spectra of compound 3a, formation of tetrazole ring was confirmed by the disappearance of nitrile peak $(-\mathrm{C} \equiv \mathrm{N})$ of compound $2 \mathbf{a}$ at $2155 \mathrm{~cm}^{-1}$ and formation of expected - $\mathrm{NH}$ stretching abortion at $3249 \mathrm{~cm}^{-1}$. Absorption band for $\mathrm{C}=\mathrm{O}$ group was absorbed at $1739 \mathrm{~cm}^{-1}$. Aliphatic and aromatic $-\mathrm{C}-\mathrm{H}$ stretching frequency was located at $2968 \mathrm{~cm}^{-1}$ and $3028 \mathrm{~cm}^{-1}$ respectively. In ${ }^{1} \mathrm{H}$ NMR spectral analysis, compound 3a displayed a singlet of one proton present on tetrazole ring at $\delta 8.21 \mathrm{ppm}$. Another singlet for two protons of methylene group exhibits at $\delta 3.94 \mathrm{ppm}$. Whereas, remaining protons appeared at their corresponding aromatic region. The IR spectra of compound $\mathbf{4 a}$ showed a strong absorption band at $3423 \mathrm{~cm}^{-1}$ for $-\mathrm{OH}$ group which is evident for the reduction of $>\mathrm{C}=\mathrm{O}$ group. It is also confirmed by the absence of carbonyl absorption band in the region 1730-1680 $\mathrm{cm}^{-1}$. In ${ }^{1} \mathrm{H}$ NMR spectra of compound $\mathbf{4 a}$ observed a singlet at $\delta 13.74 \mathrm{ppm}$ for one proton of $-\mathrm{OH}$. Another singlet is displayed at $\delta 7.98 \mathrm{ppm}$ for one proton of tetrazole ring. Similarly a triplet is detected for one proton of aliphatic $-\mathrm{CH}$ at $6.23 \mathrm{ppm}$ and a doublet for two methylene protons at 5.29 $\mathrm{ppm}$. The remaining protons were observed in their predictable aromatic region. The IR spectra of final compound 6a displayed the expected absorption band for- $\mathrm{NH}$ of tetrazole ring at 3223 $\mathrm{cm}^{-1}$. In the ${ }^{1} \mathrm{H}$ NMR spectrum analysis, compound 6a displayed a singlet resonating at $\delta 8.32$ ppm attributed to $-\mathrm{NH}$ proton of tetrazole ring. A triplet for- $\mathrm{CH}$ and a doublet for- $-\mathrm{CH}_{2}$ proton appeared at $\delta 6.35$ and $\delta 5.15 \mathrm{ppm}$ respectively. A sharp singlet at $\delta 5.86 \mathrm{ppm}$ is attributed to other methylene protons. Whereas, remaining all other aromatic protons were displayed at their 
corresponding aromatic region. Similarly in ${ }^{13} \mathrm{C}$ NMR spectra, $>\mathrm{C}=\mathrm{N}$ group of tetrazole ring appeared at160 ppm, while asymmetric carbon appeared at $104 \mathrm{ppm}$. Moreover, methylene carbons are displayed at $79 \mathrm{ppm}$ and $46 \mathrm{ppm}$. Whereas the aromatic carbons were appear in the range of 143-121 ppm. In addition, ESI-MS confirmed the identity of compound $\mathbf{6 b}$ at $m / z 381$.

\section{Biology}

All the newly synthesized target compounds (6a-p) were evaluated for their in vitro antibacterial and antifungal activities at $100 \mu \mathrm{g} / \mathrm{mL}$ concentration against S. aureus, B. subtilis as Grampositive bacteria and E.coli, P. aeruginosa and K. pneumoniae as Gram-negative bacteria. They were also screened for their in vitro antifungal potential against A. niger, A. flaus, and C. albican. Ciprofloxacin and Fluconazol were used as standard drugs for biological screening. The results of biological screening against the standard strains are recorded in Fig 2and Fig 3 which manifestly depicted the distinct sense of antibacterial and fungal strains toward the tested compounds.

\section{Antibacterial Activity:}

Regarding the antibacterial activity, the results revealed that the newly synthesized compounds displayed variable inhibitory effects on the growth of the tested Gram positive and Gram negative bacterial strains. Some of the synthesized compounds showed relatively high sensitivity against Gram positive bacterial strains namely; S. aureus and B. subtilis. In this view, compound 61 was equipotent to ciprofloxacin (MIC 3.12g/mL) against S.aureus, whereas the analogs $6 \mathrm{e}$, and $6 \mathrm{~m}$ (MIC $6.25 \mathrm{~g} / \mathrm{mL}$ ) were $50 \%$ less active than ciprofloxacin. Moreover, compound $6 \mathbf{k}$ (MIC $12.5 \mathrm{~g} / \mathrm{mL}$ ) showed $25 \%$ of the activity of ciprofloxacin against the same organism. Concerning the activity against $B$. subtilis, the best activity was displayed by compound $6 \mathbf{1}$ (MIC $6.25 \mathrm{~g} / \mathrm{mL}$ ), which represented half the potency of ciprofloxacin. On the other 
side, analog $6 \mathbf{d}, 6 \mathbf{6}, \mathbf{6 k}$, and $\mathbf{6 m}$ (MIC $12.25 \mathrm{~g} / \mathrm{mL}$ ) exhibited $25 \%$ of the potency then ciprofloxacin against the same species. On the other hand, investigation of antibacterial activity of the active compounds against the three tested Gram negative strains revealed that two analogs namely $\mathbf{6} \mathbf{d}$ and $\mathbf{6} \mathbf{l}$ were able to produce moderate growth inhibitory activity against $E$. coli (MIC $6.25 \mathrm{~g} / \mathrm{mL}$ ) which was $25 \%$ of the activity of ciprofloxacin. Whereas, compounds $6 \mathbf{e}$ and $\mathbf{6 m}$, (MIC $12.5 \mathrm{~g} / \mathrm{mL}$ ), exhibited moderate activity against the same organism. Meanwhile, the activity against $P$. aeruginosa, compound $\mathbf{6 d}, \mathbf{6 e}, \mathbf{6}$, and $\mathbf{6 m}(\mathrm{MIC} 12.5 \mathrm{~g} / \mathrm{mL})$ exhibited $50 \%$ potency as compared to ciprofloxacin. The reaming synthesized compounds were proved to be weakly sensitive against the examined strains. The screening result was exhibited in Fig 2.

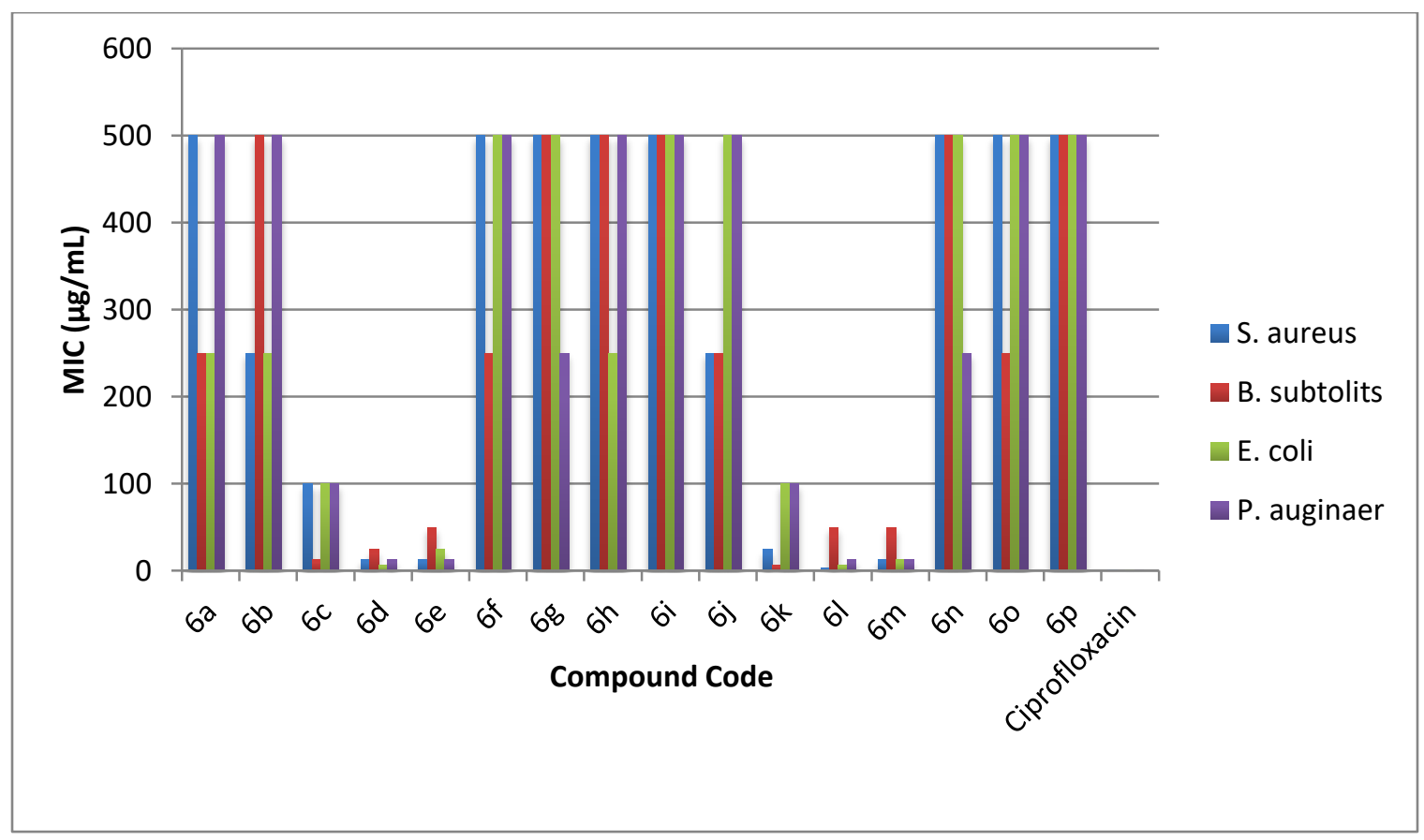

Fig 1. Graphical representation of antibacterical screening of chlorobenzyl-oxy-phenyl-ethylthio-1H-tetrazole derivatives (6a-p) 


\section{Antifungal Activity:}

The antifungal activity of the recently synthesized compounds was carried out on $A$. niger, A. flavus, and C. albicans. The screening result reveals that the compound $\mathbf{6 g}$ shows potent antifungal activity against both the three strains. Compounds $\mathbf{6 h}$ and $\mathbf{6 0}$ displayed very good potency, whereas a compound $\mathbf{6 f}, \mathbf{6 n}$, and $\mathbf{6 p}$ show good activity against A. niger, A. flavus and C. albicans. However, the remaining compounds exhibited moderate potency as compared to Fluconazol. All the antifungal screen results was displayed in Fig $\mathbf{3}$

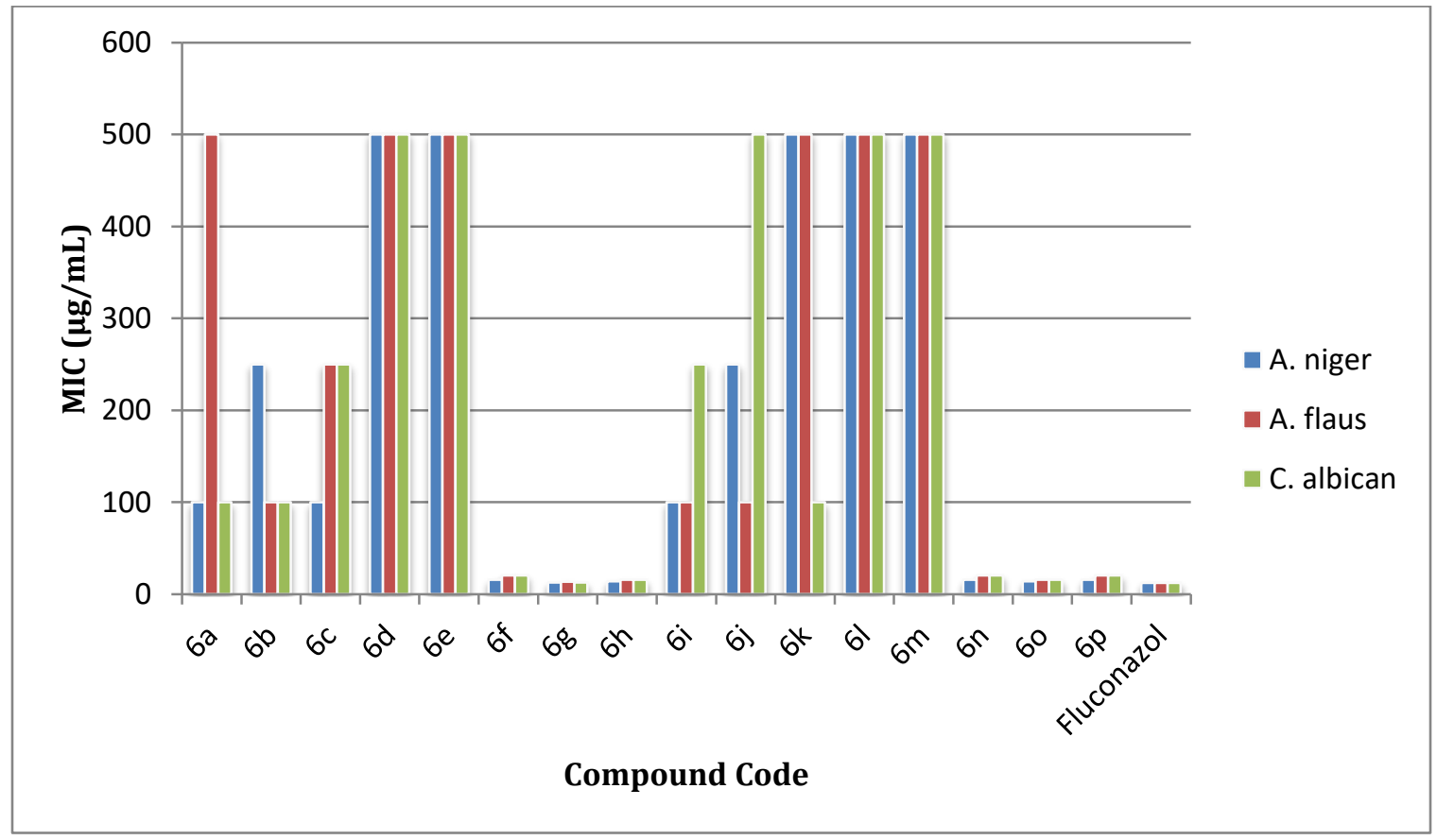

Fig 2. Graphical representation of antifungal screening of chlorobenzyl-oxy-phenyl-ethyl-thio1H-tetrazole (6a-p)

\section{Conclusions}

The research study focused on the designing and synthesis of new potentially active antibacterial and antifungal analogs on the tetrazole system. The result of in vitro pharmacological screening demonstrates that, the analogs containing electron withdrawing substituent at para position exhibit the highest antibacterial activity as compared to the standard 
drug. Whereas, compounds that have electron donating groups at para position showed promising antifungal activities. Overall, the result pharmacological result of all the synthesized novel derivatives of the tetrazole system can point out the active leads which provide a powerful incentive for the further research area.

\section{Material and Methods}

\section{Chemistry}

All melting points of newly synthesized compounds are determined in an open capillary tube and are uncorrected. Used reagents and solvents are laboratory grade and purified. IR spectra were recorded on an FTIR Perkin Elmer/Schimadzu/Bruker spectrophotometer as $\mathrm{KBr}$ pellets. ${ }^{1} \mathrm{HNMR}(400 \mathrm{MHz})$ and ${ }^{13} \mathrm{C}$ NMR (100 MHz) spectra were recorded on an Avance spectrometer (Bruker, Germany) at a $400-\mathrm{MHz}$ frequency using DMSO- $\mathrm{d}_{6}$ as a solvent with TMS as internal standard. Mass spectra were recorded on an EI-Shimadzu QP 2010 PLUS GCMS system (Shimadzu, Japan). Elemental analysis was performed on a Carlo Erba 106 PerkinElmer model 240 analyzer (Perkin-Elmer, USA). Thin layer chromatography was performed on Precoated sheets of silica gel-G (Merck, Germany) with UV lamp.

\section{General procedure for the synthesis of 1-(substituted phenyl)-2-thiocynatoethanone (2a-h)}

A mixture of potassium thiocynate $(1.00 \mathrm{~mm})$ and substituted phenacyl bromide $(1.00$ $\mathrm{mm}$ ) in PEG-400 was stirred continuously for $1-2 \mathrm{hrs}$ at $60-70^{\circ} \mathrm{C}$. Progress of the reaction was monitored by TLC and the product was separated from the crude reaction mixture in ice cold water. The separated solid was filtered simply, dried and recrystallized in aq. acetic acid to afford the pure product. 
General procedure for the synthesis of 2-((1H-tetrazol-5-yl)thio)-1-(substituted phenyl)ethanone (3a-h)

Compounds 2a-h $(1.00 \mathrm{~mm})$ and sodium azide $(1.00 \mathrm{~mm})$ were taken in PEG-400 with a catalytic amount of $\mathrm{TBAB}$. This reaction mixture was stirred at $70-80^{\circ} \mathrm{C}$ for $1-2 \mathrm{hrs}$. The reaction was monitored by TLC. After completion of the reaction, the reaction mixture was poured in ice cold water to isolate the solid product. The solid products were collected by simple filtration method, dried and recrystallized in aq. acetic acid.

2-((1H-tetrazol-5-yl)thio)-1-(4-chlorophenyl)ethanone (3a)

IR $\left(\mathrm{KBr}, \mathrm{cm}^{-1}\right): 3249(-\mathrm{NH}), 3028(\mathrm{Ar}, \mathrm{C}-\mathrm{H}), 1739(>\mathrm{C}=\mathrm{O}), 1591(>\mathrm{C}=\mathrm{N}), 1091(\mathrm{C}-\mathrm{S}-\mathrm{C})$; ${ }^{1} \mathrm{H}$ NMR (400 MHz, DMSO-d $\left.\mathrm{d}_{6}, \mathrm{TMS}, \delta, \mathrm{ppm}\right): 8.21$ (s, $1 \mathrm{H},-\mathrm{NH}$ of tetrazole), 7.95-7.47 (m, 4H, Ar-H), 3.94 (s, 2H, - $\left.\mathrm{CH}_{2}\right)$; EIMS: $254[\mathrm{M}+] ; \mathrm{C}_{9} \mathrm{H}_{7} \mathrm{ClN}_{4} \mathrm{OS}$.

2-((1H-tetrazol-5-yl)thio)-1-(4-bromophenyl)ethanone (3b)

IR $\left(\mathrm{KBr}, \mathrm{cm}^{-1}\right)$ : $3432(-\mathrm{NH}), 3012(\mathrm{Ar}, \mathrm{C}-\mathrm{H}), 1721(>\mathrm{C}=\mathrm{O}), 1612(>\mathrm{C}=\mathrm{N}) ;{ }^{1} \mathrm{H}$ NMR $(400 \mathrm{MHz}$ DMSO-d $\left.\mathrm{d}_{6}, \mathrm{TMS}, \delta, \mathrm{ppm}\right): 8.48(\mathrm{~s}, 1 \mathrm{H},-\mathrm{NH}$ of tetrazole), 8.10-7.52 (m, 4H, Ar-H), $4.24(\mathrm{~s}, 2 \mathrm{H},-$ $\mathrm{CH}_{2}$ ); EIMS: $299[\mathrm{M}+] ; \mathrm{C}_{9} \mathrm{H}_{7} \mathrm{BrN}_{4} \mathrm{OS}$.

2-((1H-tetrazol-5-yl)thio)-1-(3-nitrophenyl)ethanone (3d)

IR $\left(\mathrm{KBr}, \mathrm{cm}^{-1}\right): 3440(-\mathrm{NH}), 3011(\mathrm{Ar}, \mathrm{C}-\mathrm{H}), 1735(>\mathrm{C}=\mathrm{O}), 1605(>\mathrm{C}=\mathrm{N}), 1030(\mathrm{C}-\mathrm{S}-\mathrm{C})$;

${ }^{1} \mathrm{H}$ NMR (400 MHz, DMSO-d 6 , TMS, $\left.\delta, \mathrm{ppm}\right): 8.67$ (s, 1H, -NH of tetrazole), 8.31-7.87 (m, 4H, Ar-H), 4.05 (s, 2H, - $\mathrm{CH}_{2}$ ); EIMS: $265[\mathrm{M}+] ; \mathrm{C}_{9} \mathrm{H}_{7} \mathrm{~N}_{5} \mathrm{O}_{3} \mathrm{~S}$.

2-((1H-tetrazol-5-yl)thio)-1-(4-fluorophenyl)ethanone (3f) 
IR $\left(\mathrm{KBr}, \mathrm{cm}^{-1}\right)$ : $3418(-\mathrm{NH}), 3022(\mathrm{Ar}, \mathrm{C}-\mathrm{H}), 1730(>\mathrm{C}=\mathrm{O}), 1624(>\mathrm{C}=\mathrm{N}), 1051(\mathrm{C}-\mathrm{S}-\mathrm{C})$; ${ }^{1} \mathrm{H}$ NMR (400 MHz, DMSO-d 6 , TMS, $\left.\delta, \mathrm{ppm}\right): 8.32$ (s, 1H, -NH of tetrazole), 8.05-7.72 (m, 4H, Ar-H), $3.88\left(\mathrm{~s}, 2 \mathrm{H},-\mathrm{CH}_{2}\right)$; EIMS: $238[\mathrm{M}+] ; \mathrm{C}_{9} \mathrm{H}_{7} \mathrm{FN}_{4} \mathrm{OS}$.

2-((1H-tetrazol-5-yl)thio)-1-(3,4-dichlorophenyl)ethanone (3h)

IR $\left(\mathrm{KBr}, \mathrm{cm}^{-1}\right): 3449(-\mathrm{NH}), 3032(\mathrm{Ar}, \mathrm{C}-\mathrm{H}), 1732(>\mathrm{C}=\mathrm{O}), 1611(>\mathrm{C}=\mathrm{N}), 1014(\mathrm{C}-\mathrm{S}-\mathrm{C})$; ${ }^{1} \mathrm{H}$ NMR (400 MHz, DMSO-d 6 , TMS, $\left.\delta, \mathrm{ppm}\right): 8.82$ (s, $1 \mathrm{H},-\mathrm{NH}$ of tetrazole), 8.18-7.87 (m, 3H, Ar-H), 4.57 (s, 2H, - $\mathrm{CH}_{2}$ ); EIMS: $289[\mathrm{M}+] ; \mathrm{C}_{9} \mathrm{H}_{6} \mathrm{Cl}_{2} \mathrm{~N}_{4} \mathrm{OS}$.

General procedure for the synthesis of 2-((1H-tetrazol-5-yl)thio)-1-substitutedphenylethanol $(4 a-h)$

Compounds 3a-h $(1.00 \mathrm{~mm})$ in PEG-400 was stirred at room temperature in presence of a catalytic amount of sodium borohydrate $(0.25 \mathrm{~mm})$ for $30 \mathrm{~min}$. After completion of the reaction (monitored by TLC), the reaction mixture stands for $1 \mathrm{hr}$ at room temperature and is poured in ice cold water. The separated product was filtered, dried wash with water and recrystallized from aq. acetic acid.

2-((1H-tetrazol-5-yl)thio)-1-(4-chlorophenyl)ethanol (4a)

IR (KBr, cm $\left.{ }^{-1}\right): 3423(-\mathrm{OH}), 3217(-\mathrm{NH}), 1615(>\mathrm{C}=\mathrm{N}), 1091(\mathrm{C}-\mathrm{O}-\mathrm{C}) ;{ }^{1} \mathrm{H}$ NMR $(400 \mathrm{MHz}$, DMSO-d $\left.\mathrm{d}_{6}, \mathrm{TMS}, \delta, \mathrm{ppm}\right): 13.74(\mathrm{~s}, 1 \mathrm{H},-\mathrm{OH}), 7.98(\mathrm{~s}, 1 \mathrm{H},-\mathrm{NH}$ of tetrazole), $7.86-7.46(\mathrm{~m}, 4 \mathrm{H}$, Ar-H), $6.23(\mathrm{t}, 1 \mathrm{H},-\mathrm{CH}), 5.29\left(\mathrm{~d}, 2 \mathrm{H},-\mathrm{CH}_{2}\right)$.

\section{2-((1H-tetrazol-5-yl)thio)-1-(4-bromophenyl)ethanol (4b)}

IR (KBr, cm $\left.{ }^{-1}\right): 3457(-\mathrm{OH}), 3192(-\mathrm{NH}), 1622(>\mathrm{C}=\mathrm{N}), 1108(\mathrm{C}-\mathrm{O}-\mathrm{C}) ;{ }^{1} \mathrm{H}$ NMR $(400 \mathrm{MHz}$, DMSO-d $\left.\mathrm{d}_{6}, \mathrm{TMS}, \delta, \mathrm{ppm}\right): 13.88(\mathrm{~s}, 1 \mathrm{H},-\mathrm{OH}), 8.32(\mathrm{~s}, 1 \mathrm{H},-\mathrm{NH}$ of tetrazole), $8.11-7.87(\mathrm{~m}, 4 \mathrm{H}$, Ar-H), 6.37 (t, 1H, -CH), $5.12\left(\mathrm{~d}, 2 \mathrm{H},-\mathrm{CH}_{2}\right)$. 


\section{2-((1H-tetrazol-5-yl)thio)-1-(3-nitrophenyl)ethanol (4d)}

IR (KBr, cm $\left.{ }^{-1}\right): 3528(-\mathrm{OH}), 3196(-\mathrm{NH}), 1620(>\mathrm{C}=\mathrm{N}), 1128(\mathrm{C}-\mathrm{O}-\mathrm{C}) ;{ }^{1} \mathrm{H}$ NMR (400 MHz, DMSO-d 6 , TMS, $\delta, \mathrm{ppm}): 13.87(\mathrm{~s}, 1 \mathrm{H},-\mathrm{OH}), 8.56(\mathrm{~s}, 1 \mathrm{H},-\mathrm{NH}$ of tetrazole), $8.28-7.89(\mathrm{~m}, 4 \mathrm{H}$, Ar-H), $6.42(\mathrm{t}, 1 \mathrm{H},-\mathrm{CH}), 5.14\left(\mathrm{~d}, 2 \mathrm{H},-\mathrm{CH}_{2}\right)$.

2-((1H-tetrazol-5-yl)thio)-1-(4-fluorophenyl)ethanol (4f)

IR (KBr, cm $\left.{ }^{-1}\right): 3487(-\mathrm{OH}), 3174(-\mathrm{NH}), 1631(>\mathrm{C}=\mathrm{N}), 1132(\mathrm{C}-\mathrm{O}-\mathrm{C}) ;{ }^{1} \mathrm{H}$ NMR $(400 \mathrm{MHz}$, DMSO-d 6 , TMS, $\delta, \mathrm{ppm}): 13.17(\mathrm{~s}, 1 \mathrm{H},-\mathrm{OH}), 8.38(\mathrm{~s}, 1 \mathrm{H},-\mathrm{NH}$ of tetrazole), $8.17-7.81(\mathrm{~m}, 4 \mathrm{H}$, Ar-H), $6.46(\mathrm{t}, 1 \mathrm{H},-\mathrm{CH}), 5.30\left(\mathrm{~d}, 2 \mathrm{H},-\mathrm{CH}_{2}\right)$.

\section{2-((1H-tetrazol-5-yl)thio)-1-(3,4-dichlorophenyl)ethanol (4h)}

IR $\left(\mathrm{KBr}, \mathrm{cm}^{-1}\right): 3413(-\mathrm{OH}), 3237(-\mathrm{NH}), 1625(>\mathrm{C}=\mathrm{N}), 1128(\mathrm{C}-\mathrm{O}-\mathrm{C}) ;{ }^{1} \mathrm{H}$ NMR (400 MHz, DMSO-d 6 , TMS, $\delta, \mathrm{ppm}): 13.87(\mathrm{~s}, 1 \mathrm{H},-\mathrm{OH}), 8.66(\mathrm{~s}, 1 \mathrm{H},-\mathrm{NH}$ of tetrazole), $8.43-7.96(\mathrm{~m}, 4 \mathrm{H}$, Ar-H), $6.22(\mathrm{t}, 1 \mathrm{H},-\mathrm{CH}), 5.30\left(\mathrm{~d}, 2 \mathrm{H},-\mathrm{CH}_{2}\right)$.

General procedure for the synthesis of 5-((2-((4-chlorobenzyl)oxy)-2-(4chlorophenyl)ethyl)thio)-1H-tetrazole (6a-p)

An equimolar mixture of compounds $\mathbf{4 a - h}(1.00$ mmole $)$ and substituted chlobenzylchloride $(1.00$ mmole $)$ were stirred continuously in presence of a catalytic amount of Bleaching Earth Clay (pH-12.5, $10 \mathrm{wt} \%$ ) as a heterogeneous catalyst to attain basic media in PEG-400 for $1 \mathrm{hr}$ at $70-80^{\circ} \mathrm{C}$. After completion of reaction monitored by TLC the reaction mixture was cooled at room temperature and worked up with ice cold water. After filtration the separated product was washed with hot water, dried and recrystallized from aq. acetic acid to obtain a pure final product. 


\section{5-((2-((4-chlorobenzyl)oxy)-2-(4-chlorophenyl)ethyl)thio)-1H-tetrazole (6a)}

M.P. $167-169^{\circ} \mathrm{C}$; Yield: 86\%; IR (KBr, cm $\left.{ }^{-1}\right)$ : $3215(-\mathrm{NH}), 3068$ (aromatic-C-H), $1617(>\mathrm{C}=\mathrm{N})$,

1073 (C-O-C); ${ }^{1} \mathrm{H}$ NMR (400 MHz, DMSO-d 6 , TMS, $\delta$, ppm): 8.23 (s, 1H, -NH of tetrazole), 7.86-7.46 (m, 8H, Ar-H), $6.35(\mathrm{~d}, 1 \mathrm{H},-\mathrm{CH}), 5.86\left(\mathrm{~s}, 2 \mathrm{H},-\mathrm{CH}_{2}\right), 5.15\left(\mathrm{t}, 2 \mathrm{H},-\mathrm{CH}_{2}\right) ;{ }^{13} \mathrm{C}$ NMR (100 MHz, DMSO-d6, TMS, $\delta$, ppm); 160.41, 143.12, 138.16, 136.86, 135.19, 132.51, 131.30, 130.16, 129.81, 129.31, 128.92, 121.46, 104.43, 79.19, 46.86; EIMS: 380 [M+]; Elemental Analysis: Caculated (found) for $\mathrm{C}_{16} \mathrm{H}_{14} \mathrm{Cl}_{2} \mathrm{~N}_{4} \mathrm{OS}$ : \% C, 50.40 (50.42); H, 3.70 (3.73); N, 14.69 (14.67); S, $8.41(8.43)$.

5-((2-((4-chlorobenzyl)oxy)-2-(4-nitrophenyl)ethyl)thio)-1H-tetrazole (6c)

M.P. $182-184^{\circ} \mathrm{C}$; Yield: 92\%; IR $\left(\mathrm{KBr}, \mathrm{cm}^{-1}\right)$ : $3262(-\mathrm{NH}), 3120$ (aromatic-C-H), $1622(>\mathrm{C}=\mathrm{N})$, 1112 (C-O-C); ${ }^{1} \mathrm{H}$ NMR (400 MHz, DMSO-d 6 , TMS, $\left.\delta, \mathrm{ppm}\right): 8.73$ (s, 1H, -NH of tetrazole), 8.37-7.35 (m, 8H, Ar-H), $6.43(\mathrm{t}, 1 \mathrm{H},-\mathrm{CH}), 5.72\left(\mathrm{~s}, 2 \mathrm{H},-\mathrm{CH}_{2}\right), 5.22\left(\mathrm{~d}, 2 \mathrm{H},-\mathrm{CH}_{2}\right) ;{ }^{13} \mathrm{C}$ NMR (100 MHz, DMSO-d6, TMS, $\delta$, ppm); 164.62, 146.89, 140.84, 138.18, 136.37, 135.42, 134.98, 133.72, 131.46, 130.61, 129.28, 126.55, 100.49, 71.76, 43.68; EIMS: 391 [M+]; Elemental Analysis: Calculated (found) for $\mathrm{C}_{16} \mathrm{H}_{14} \mathrm{ClN}_{5} \mathrm{O}_{3} \mathrm{~S}: \%$ C, 49.04 (49.01); H, 3.60 (3.62); N, 17.87 (17.85); S, 8.18 (8.20).

\section{5-((2-((4-chlorobenzyl)oxy)-2-(3-nitrophenyl)ethyl)thio)-1H-tetrazole (6d)}

M.P. $177-179^{\circ} \mathrm{C}$; Yield: 94\%; IR (KBr, $\left.\mathrm{cm}^{-1}\right): 3289(-\mathrm{NH}), 2977$ (aromatic-C-H), $1639(>\mathrm{C}=\mathrm{N})$, 1127 (C-O-C); ${ }^{1} \mathrm{H}$ NMR (400 MHz, DMSO-d 6 , TMS, $\left.\delta, p p m\right): 8.47$ (s, 1H, -NH of tetrazole), 8.20-7.24 (m, 8H, Ar-H), $6.93(\mathrm{t}, 1 \mathrm{H},-\mathrm{CH}), 5.98\left(\mathrm{~s}, 2 \mathrm{H},-\mathrm{CH}_{2}\right), 5.37\left(\mathrm{~d}, 2 \mathrm{H},-\mathrm{CH}_{2}\right) ;{ }^{13} \mathrm{C}$ NMR (100 MHz, DMSO-d6, TMS, $\delta$, ppm); 155.45, 147.75, 138.47, 137.64 132.45, 129.94, 129.86, 129.71, 128.24, 127.69, 124.04, 123.95, 94.32, 80.24, 47.86; EIMS: 391 [M+]; Elemental 
Analysis: Calculated (found) for $\mathrm{C}_{16} \mathrm{H}_{14} \mathrm{ClN}_{5} \mathrm{O}_{3} \mathrm{~S}: \%$ C, 49.04 (49.01); H, 3.60 (3.62); N, 17.87 (17.90); S, 8.18 (8.20).

5-((2-((4-chlorobenzyl)oxy)-2-(p-tolyl)ethyl)thio)-1H-tetrazole (6g)

M.P. $163-165^{\circ} \mathrm{C}$; Yield: 91\%; IR (KBr, $\left.\mathrm{cm}^{-1}\right)$ : $3187(-\mathrm{NH}), 3066$ (Aromatic-C-H), $1608(>\mathrm{C}=\mathrm{N})$, 1124 (C-O-C); ${ }^{1} \mathrm{H}$ NMR (400 MHz, DMSO-d 6 , TMS, $\left.\delta, p p m\right): 8.67$ (s, 1H, -NH of tetrazole), 8.78-7.29 (m, 8H, Ar-H), $6.13\left(\mathrm{t}, 2 \mathrm{H},-\mathrm{CH}_{2}\right), 5.69$ (s, 1H, -CH), 4.67 (d, 2H, - $\left.\mathrm{CH}_{2}\right), 2.29$ (s, 3H, $\left.\mathrm{CH}_{3}\right) ;{ }^{13} \mathrm{C}$ NMR (100 MHz, DMSO-d6, TMS, $\left.\delta, \mathrm{ppm}\right) ; 161.83,141.73,138.57,136.31,135.28$, $130.91,130.33,129.42,122.89,128.01,126.94,126.13,98.36,78.53,46.29,22.78$; EIMS: 360 $[\mathrm{M}+]$; Elemental Analysis: Calculated (found) for $\mathrm{C}_{17} \mathrm{H}_{17} \mathrm{ClN}_{4} \mathrm{OS}$ : \% C, 56.58 (56.56); H, 4.75 (4.78); N, 15.53 (15.52); S, 8.89 (8.87).

\section{5-((2-((2,4-dichlorobenzyl)oxy)-2-(4-nitrophenyl)ethyl)thio)-1H-tetrazole (6j)}

M.P. $187-189^{\circ} \mathrm{C}$; Yield: 90\%; IR (KBr, $\left.\mathrm{cm}^{-1}\right): 3171(-\mathrm{NH}), 3129$ (Aromatic-C-H), $1597(>\mathrm{C}=\mathrm{N})$, 1079 (C-O-C); ${ }^{1} \mathrm{H}$ NMR (400 MHz, DMSO-d 6 , TMS, $\left.\delta, \mathrm{ppm}\right): 8.29$ (s, 1H, -NH of tetrazole), 8.16-7.42 (m, 8H, Ar-H), $6.54(\mathrm{t}, 1 \mathrm{H},-\mathrm{CH}), 5.71\left(\mathrm{~s}, 2 \mathrm{H},-\mathrm{CH}_{2}\right), 5.29\left(\mathrm{~d}, 2 \mathrm{H},-\mathrm{CH}_{2}\right) ;{ }^{13} \mathrm{C}$ NMR (100 MHz, DMSO-d6, TMS, $\delta$, ppm); 158.73, 147.62, 136.81, 135.19, 134.57, 133.66, 132.95, 129.36, 128.47, 128.92, 126.63, 124.52, 96.39, 71.98, 43.74; EIMS: 425 [M+]; Elemental Analysis: Calculated (found) for $\mathrm{C}_{16} \mathrm{H}_{13} \mathrm{Cl}_{2} \mathrm{~N}_{5} \mathrm{OS}$ : \% C, 45.08 (45.05); H, 3.07 (3.09); N, 16.43 (16.45); S, 7.52 (7.54).

\section{5-((2-((2,4-dichlorobenzyl)oxy)-2-phenylethyl)thio)-1H-tetrazole (6m)}

M.P. $165-167^{\circ} \mathrm{C}$; Yield: $85 \%$; IR $\left(\mathrm{KBr}, \mathrm{cm}^{-1}\right): 3159(-\mathrm{NH}), 3143$ (Aromatic-C-H), $1588(>\mathrm{C}=\mathrm{N})$, 1069 (C-O-C); ${ }^{1} \mathrm{H}$ NMR (400 MHz, DMSO-d 6 , TMS, $\delta$, ppm): 8.67 (s, 1H, -NH of tetrazole), 8.47-7.72 (m, 9H, Ar-H), $6.58(\mathrm{t}, 1 \mathrm{H},-\mathrm{CH}), 6.08\left(\mathrm{~s}, 2 \mathrm{H},-\mathrm{CH}_{2}\right), 5.78\left(\mathrm{~d}, 2 \mathrm{H},-\mathrm{CH}_{2}\right) ;{ }^{13} \mathrm{C}$ NMR (100 MHz, DMSO-d6, TMS, $\delta$, ppm); 159.82, 142.53, 137.86, 135.28, 134.93, 131.47, 130.61, 
129.77, 129.03, 128.94, 127.84, 127.06, 120.24, 102.33, 82.47, 44.80; EIMS: $380[\mathrm{M}+]$;

Elemental Analysis: Calculated (found) for $\mathrm{C}_{16} \mathrm{H}_{14} \mathrm{Cl}_{2} \mathrm{~N}_{4} \mathrm{OS}$ : \% C, 50.40 (50.37); H, 3.70 (3.67);

N, 14.69 (14.66); S, $8.41(8.39)$.

\section{Biology}

All the newly synthesized target compounds (6a-p) were evaluated for their in vitro antibacterial and antifungal activities at $100 \mu \mathrm{g} / \mathrm{mL}$ concentration against Staphylococcus aureus (MTCC), Bacillis subtilis (MTCC) as examples of Gram-positive bacteria and Esherichia coli (MTCC), and Klebsiella pneumoniae (MTCC) as examples of Gram-negative bacteria. They were also screened for their in vitro antifungal potential against Aspergillus niger (MTCC282),Aspergillus flaus (MTCC 3008), and Candida albican (MTCC 227). The evolution of preliminary antibacterial and antifungal activities was determined by agar-diffusion method [54] using a 1 $\mathrm{cm}$ microplate well. Ciprofloxacin and Fluconazol were used as standard drugs for biological screening. The results of biological screening against the standard strains are recorded in Fig. 1 and Fig. 2 which manifestly depicted the distinct sense of antibacterial and fungal strains toward the tested compounds.

\section{References:}

1. Zhang L, Peng X M, Damu GLV, Geng RX, Zhou CH. Comprehensive Review in Current Developments of Imidazole-Based Medicinal Chemistry. Med Res Rev. 2014; 34(2): 340-37. https://doi.org/10.1002/med.21290

2. Peng XM, Damu GLV, Zhou CH. Current Developments of Coumarin Compounds in Medicinal Chemistry. Curr Pharm Des. 2013; 19: 3884-30. https://doi.org/10.2174/1381612811319210013 
3. Scheffler RJ, Colmer S, Tynan H, Demain A L, Gullo V P. Antimicrobials, drug discovery, and genome mining. Appl Microbiol Biotechnol. 2013; 97: 969-78. https://doi.org/10.1007/s00253-012-4609-8

4. Constantinos GN, Ting Z, Alexander D. Tetrazoles via Multicomponent Reactions. Chem Rev. 2019; 119 (3): 1970-42. https://doi.org/10.1021/acs.chemrev.8b00564

5. Jaroslav R, Katerina V, Alexandr H. Synthesis and Functionalization of 5-Substituted Tetrazoles. Eur J Org Chem. 2012; 12: 6101-18. https://doi.org/10.1002/ejoc.201200469

6. Wei CX, Bian M, Gong GH. Tetrazolium Compounds: Synthesis and Applications in Medicine. Molecules. 2015; 20(4): 5528- 53. https://doi.org/10.3390/molecules20045528

7. Frija LMT, Ismael A, Cristiano MLS. Photochemical Transformations of Tetrazole Derivatives: Applications in Organic Synthesis. Molecules. 2010; 15(5): 3757-74. https://dx.doi.org/10.3390\%2Fmolecules 15053757

8. Shmatova OI, Nenajdenko VG, Synthesis of TetrazoleDerived Organocatalysts via Azido-Ugi Reaction with Cyclic Ketimines J Org Chem. 2013; 78(18): 9214-22. https://doi.org/10.1021/jo401428q

9. Ostrovskii VA, Trifonov RE, Popova EA. Medicinal chemistry of tetrazoles Russ Chem Bull. 2012; 61: 768-80. https://doi.org/10.1007/s11172-012-0108-4

10. Sherif AF, Rostom, Hayam MA. Ashour HA et al. Azole antimicrobial pharmacophorebased tetrazoles: Synthesis and biological evaluation as potential antimicrobial and anticonvulsant agents. Bioorg Med Chem. 2009;17(6): 2410-22. https://doi.org/10.1016/j.bmc.2009.02.004

11. Feng G, Jiaqi X, Gang H. Current scenario of tetrazole hybrids for antibacterial activity. Eur J Med Chem. 2019; 184: 111744. https://doi.org/10.1016/j.ejmech.2019.111744 
12. Daniel S, Michał A. Dobrowolski, Piotr R, Anna B, Joanna S, Michał K et al. Design and synthesis of novel 1H-tetrazol-5-amine based potent antimicrobial agents: DNA topoisomerase IV and gyrase affinity evaluation supported by molecular docking studies. Eur J Med Chem., 2018; 156(5): 631-40. https://doi.org/10.1016/j.ejmech.2018.07.041

13. Kritchenkov AS, Lipkan NA, Kurliuk AV, Shakola TV, et al Synthesis and Antibacterial Activity of Chitin Tetrazole Derivatives. Pharm Chem J. 2020; 54: 138-41. https://doi.org/10.1007/s11094-020-02180-4

14. Edyta LC, Anna K, Malgorzata G, Miroslawa K, Monika S, Synthesis of tetrazole derivatives bearing pyrrolidine scaffold and evaluation of their antifungal activity against Candida albicans. Eur J Med Chem. 2019; 164(15): 106-20. https://doi.org/10.1016/j.ejmech.2018.12.044

15. Shaikh SK, Kamble RR, Somagond SM,. Devarajegowda HC, Dixit SR, Joshi SD. Tetrazolylmethyl quinolines: Design, docking studies, synthesis, anticancer and antifungal analyses. Eur J Med Chem. 2017; 128(10): 258-73. https://doi.org/10.1016/j.ejmech.2017.01.043

16. Mikolaichuk OV, Zarubaev VV, Muryleva AA, Esaulkova YL, Spasibenko DV, et al. Synthesis, structure, and antiviral properties of novel 2-adamantyl-5-aryl-2H-tetrazoles. Chem Het Comp. 2021; 57: 442-47. https://doi.org/10.1007/s10593-021-02931-5

17. Gao C, Chang L, Xu Z, Yan XF, Ding C, Zhao F, Wu X, Feng LS. Recent advances of tetrazole derivatives as potential anti-tubercular and anti-malarial agents. Eur $\mathbf{J}$ Med Chem. 2019; 163 (1): 404-12. https://doi.org/10.1016/j.ejmech.2018.12.001 
18. Zhang S, Xu Z, Gao C, Ren QC, Le C, Lv ZS, Feng LS, Triazole derivatives and their anti-tubercular activity. Eur J Med Chem. 2017; 138(29): 501-13. https://doi.org/10.1016/j.ejmech.2017.06.051

19. Elena AP, Aleksandra VP, Rostislav ET. Tetrazole Derivatives as Promising Anticancer Agents. Anticancer Agents Med Chem. 2018; 17(14):1856-68. https://doi.org/10.2174/1871520617666170327143148

20. Kohler SC, Vahdati S, Scholz MS, Wiese M. Structure activity relationships, multidrug resistance reversal and selectivity of heteroarylphenyl ABCG2 inhibitors. Eur J Med Chem. 2018; 146(25): 483-500. https://doi.org/10.1016/j.ejmech.2018.01.012

21. Kalaria PN, Karad SC, Raval DK. A review on diverse heterocyclic compounds as the privileged scaffolds in antimalarial drug discovery. Eur J Med Chem. 2018; 158(5): 91736. https://doi.org/10.1016/j.ejmech.2018.08.040

22. Pandey S, Agarwal P, Srivastava K, Rajkumar S, et al. Synthesis and bioevaluation of novel 4-aminoquinoline-tetrazole derivatives as potent antimalarial agents. Eur J Med Chem. 2013; 66: 69-81. https://doi.org/10.1016/j.ejmech.2013.05.023

23. Li Y, Pasunooti KK, Li RJ, Liu W, Head SA, Shi WQ, Liu JO. Novel TetrazoleContaining Analogues of Itraconazole as Potent Antiangiogenic Agents with Reduced Cytochrome P450 3A4 Inhibition. J Med Chem. 2018; 61(24): 11158-68. https://doi.org/10.1021/acs.jmedchem.8b01252

24. Li Y, Pasunooti KK, Peng H, Li RJ, Shi WQ, et al. Design and Synthesis of Tetrazoleand Pyridine-Containing Itraconazole Analogs as Potent Angiogenesis Inhibitors. Med Chem Lett. 2020; 11(6):1111-17.https://dx.doi.org/10.1021\%2Facsmedchemlett.9b00438 
25. Esirden I, Tanc M, Supuran CT, Kaya M, Microwave assisted synthesis of novel tetrazole/sulfonamide derivatives based on octahydroacridine, xanthene and chromene skeletons as inhibitors of the carbonic anhydrases isoforms I, II, IV and VII. Bioorg Med Chem Lett. 2017;27(1): 86-89. https://doi.org/10.1016/j.bmcl.2016.11.028

26. Lamie PF, Philoppes JN, Azouz AA, Safwatc NM. Novel tetrazole and cyanamide derivatives as inhibitors of cyclooxygenase-2 enzyme: design, synthesis, antiinflammatory evaluation, ulcerogenic liability and docking study. J Enzyme Inhib Med Chem. 2017; 32(1): 805-20. https://doi.org/10.1080/14756366.2017.1326110

27. Zhan P, Li Z, Liu X, Clercq ED. Sulfanyltriazole/tetrazoles: a promising class of HIV-1 NNRTIs. Mini Rev Med Chem. 2009; 9(8):1014-23. https://doi.org/10.2174/138955709788681618

28. Zhan P, Liu H, Liu X, Wang Y, Pannecouque C, Witvrouw M, Clercq ED. Synthesis and anti-HIV activity evaluation of novel N'-arylidene-2-[1-(naphthalen-1-yl)-1H-tetrazol-5ylthio]acetohydrazides. Med Chem Res. 2010; 19: 652-63. https://doi.org/10.1007/s00044-009-9220-X

29. Rajasekaran A, Thampi PP. Synthesis and analgesic evaluation of some 5-[ $\beta$-(10phenothiazinyl)ethyl]-1-(acyl)-1,2,3,4-tetrazoles. Eur J Med Chem. 2004; 39(3): 273-79. https://doi.org/10.1016/j.ejmech.2003.11.016

30. Kharb R, Sharma PC, Yar MS. Pharmacological significance of triazole scaffold. J Enzy InhiB Med Chem. 2011; 26(1): 1-21. https://doi.org/10.3109/14756360903524304

31. Kushwaha O, Fatima S, Upadhyay A, Gupta S, Bhagwati S, Baghel T, Siddiqi MI, Nazir A, Sashidhara KV. Synthesis, biological evaluation and molecular dynamic simulations 
of novel Benzofuran-tetrazole derivatives as potential agents against Alzheimer's disease. Bioorg Med Chem Lett. 2019; 29(1) 66-72. https://doi.org/10.1016/j.bmcl.2018.11.005

32. Hassan AEA, Abou-Elkhair RAI, Parker WB, Allan PW, Secrist JA, 6-Methylpurine derived sugar modified nucleosides: Synthesis and evaluation of their substrate activity with purine nucleoside phosphorylases. Bioorg Chem. 2016; 65: 9-16. https://doi.org/10.1016/j.bioorg.2015.12.006

33. Cardoso CS, Silva DPB, Silva DM, Florentino IF, et al. Mechanisms involved in the antinociceptive and anti-inflammatory effects of a new triazole derivative: 5-[1-(4fluorophenyl)-1H-1,2,3-triazol-4-yl]-1H-tetrazole (LQFM-096). Inflammopharmacol. 2020; 28: 877-92. https://doi.org/10.1007/s10787-020-00685-8

34. Rajasekaran A, Thampi PP. Synthesis and antinociceptive activity of some substituted-

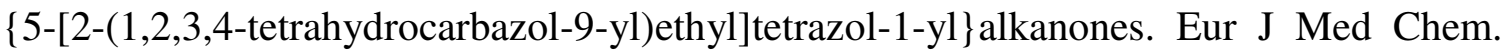
2005; 40(12):1359-64. https://doi.org/10.1016/j.ejmech.2005.07.013

35. Bian M, Deng XQ, Gona GH, Wei CX, Quan ZS. Synthesis and anticonvulsant activity evaluation of 6-substituted-[1,2,4]triazolo[3,4-a](tetrazolo[5,1-a])phthalazine derivatives. J Enzy Inhib Med Chem, 2013; 28(4): 792-800. https://doi.org/10.3109/14756366.2012.684052

36. Rajasekaran A, Murugesan S, AnandaRajagopal K. Antibacterial, antifungal and anticonvulsant evaluation of novel newly synthesized 1-[2-(1H-tetrazol-5-yl)ethyl]-1Hbenzo[d][1,2,3]triazoles. Arch Pharm Res. 2006; 29(7): 535-40. https://doi.org/10.1007/BF02969261

37. Liljebris C, Larsen SD, Ogg D, Palazuk BJ, Bleasdale JE. Investigation of Potential Bioisosteric Replacements for the Carboxyl Groups of Peptidomimetic Inhibitors of 
Protein Tyrosine Phosphatase 1B: Identification of a Tetrazole-Containing Inhibitor with Cellular Activity. J Med Chem. 2002; 45(9): 1785-1798. https://doi.org/10.1021/jm011100y

38. Kozikowski A P, Zhang J. Nan F, Petukhov P A, Grajkowska E, Wroblewski J T, Yamamoto T, Bzdega T, Wroblewska B, Neale J, Synthesis of Urea-Based Inhibitors as Active Site Probes of Glutamate Carboxypeptidase II: Efficacy as Analgesic Agents. J Med Chem. 2004; 47(7): 1729-1738. https://doi.org/10.1021/jm0306226

39. Myznikov L V, Hrabalek A, Koldobskii G I, Drugs in the tetrazole series. (Review) Chem. Heterocycl Compd. 2007; 43(1): 1-9. https://doi.org/10.1007/s10593-007-0001-5

40. Koldobskii GI, Ostrovskii VA, Tetrazoles Usp. Khim., 1994; 63(10): 847-865.

41. Fischer D, Klapötke TM, Stierstorfer J, Angew. 1,5-Di(nitramino)tetrazole: High Sensitivity and Superior Explosive Performance. Chem Int Ed. 2015; 54(35): 1029910302. https://doi.org/10.1002/anie.201502919

42. Klapotke T M, Stierstorfer J, Green Energetic Materials, John Wiley \& Sons, Ltd, 2014.

43. Dippold AA, Izsak D, Klapötke TM, Pflüger C, Combining the Advantages of Tetrazoles and 1,2,3-Triazoles: 4,5-Bis(tetrazol-5-yl)-1,2,3-triazole, 4,5-Bis(1hydroxytetrazol-5-yl)-1,2,3-triazole, and their Energetic Derivatives. Chem Eur J. 2016; 22(5): 1768-1778. https://doi.org/10.1002/chem.201504624

44. Yu Z, Ho LY, Wang Z, Lin Q, Discovery of new photoactivatable diaryltetrazoles for photoclick chemistry via 'scaffold hopping'. Bioorg Med Chem Lett. 2011; 21(17): 50336. https://dx.doi.org/10.1016\%2Fj.bmcl.2011.04.087

45. Wang Y, Lin Q, Synthesis and Evaluation of Photoreactive Tetrazole Amino Acids. Org Lett. 2009; 11(16): 3570-3573. https://doi.org/10.1021/o1901300h 
46. Wang Y, Hu WJ, Song W, Lim RKV, Lin Q. Org. Lett. 2008; 10(17): 3725-3728. https://doi.org/10.1021/ol801350r

47. Wurzenberger MHH, Gruhne MS, Lommel M, Szimhardt N, Klapotke TM, Stierstorfer J. Comparison of 1-Ethyl-5H-tetrazole and 1-Azidoethyl-5H-tetrazole as Ligands in Energetic Transition Metal Complexes. Chem Asian J. 2019; 14(11): 2018-2028. https://doi.org/10.1002/asia.201900269

48. Yang L, Shen Y, Chen Y, Pan X, Wang X, A novel octamolybdate-based metal-organic complex constructed from a bis(tetrazole)-functionalized thioether ligand and an Anderson-type polyoxometalate. Inorg Chem Comm. 2019; 108: 107493. https://doi.org/10.1016/j.inoche.2019.107493

49. Ambhore AN, Kamble SS, Kadam SN, Kamble RD, Hebade MJ, Hese SV, Gaikwad MV, Mesharam RJ, Gacche RN, Dawane BS, Design, synthesis and in silico study of pyridine based 1, 3, 4-oxadiazole embedded hydrazinecarbothioamide derivatives as potent anti-tubercular agent. Comp Bio Chem. 2019; 80; 54-65. https://doi.org/10.1016/j.compbiolchem.2019.03.002

50. Ambhore AN, Surywanshi VD, Kamble RD, Hese SV, Mogale PP, Kadam SS, Dawane BS, Synthesis and biological investigation of isoxazolo $[4,5-e][1,2,4]$ triazine derivatives. Der Phar Chem. 2015; 7(4); 278-283. http://derpharmachemica.com/archive.html

51. Kadam SN, Ambhore AN, Kamble RD, Wakhradkar MG, Gavhane PD, Gaikwad MV, Gunturu KC, Dawane BS, Metal-free efficient thiolation of C ( $\operatorname{sp} 2$ ) functionalization via in situ-generated NHTS for the synthesis of novel sulfenylated 2-aminothiazole and imidazothiazole. N J Chem. 2021; 45; 4632-4637. https://doi.org/10.1039/D0NJ05904H 
52. Gavhane PD, Kadam SN, Ambhore AN, Dawane BS, Silica-supported sodium carbonate: an efficient heterogeneous catalyst for the synthesis of new thiazolopyrimidine derivatives. Res Chem Inter. 2021; 47; 3999-4011. https://doi.org/10.1007/s11164-021$\underline{04508-5}$

53. Kadam SN, Ambhore AN, Hebade MJ, Kamble RD, Hese SV, Gaikwad MV, Gavhane PD, Dawane BS, Metal-Free One-Pot Chemoselective thiocyanation of imidazothiazoles and 2-aminothiazoles with in situ generated N-thiocyanatosuccinimide. Synlett. 2018; 29; 1902-1908. http://doi.org/10.1055/s-0037-1609553

54. Hese SV, Kamble RD, Mogale PP, Kadam SS, Hebade MJ, Ambhore An, Dawane BS, Green synthesis and antimicrobial evaluation of pyrido [1,2-a] pyrimidine-3-carbonitrile $\begin{array}{llllll}\text { derivatives. } & \text { Der Pharma } & \text { Chem. 2015; } 79-256 .\end{array}$ http://derpharmachemica.com/archive.html 


\section{Supplementary Files}

This is a list of supplementary files associated with this preprint. Click to download.

- Figers.docx

- SupplimentaryData.docx 